\title{
Letters
}

\section{Photoacoustic Imaging of Multiple Targets Using Gold Nanorods}

Pai-Chi Li, Chen-Wei Wei, Chao-Kang Liao, Cheng-Dah Chen, Kuei-Chen Pao,

Churng-Ren Chris Wang, Ya-Na Wu, and Dar-Bin Shieh

\begin{abstract}
Photoacoustic (PA) imaging has been used mainly for anatomical and functional imaging. Although functionalized nanoparticles also have been developed for PA molecular imaging, only single targeting has been demonstrated. In this study, PA imaging of multiple targets using gold nanorods is demonstrated experimentally using HER2 and CXCR4 as target molecules. The two corresponding monoclonal antibodies were conjugated to two types of gold nanorod with different aspect ratios. Gold nanorods with mean aspect ratios of 5.9 and 3.7 exhibited peak optical absorptions at 1000 and $785 \mathrm{~nm}$, respectively. Appropriate selection of laser irradiation wavelength enhances PA signals by 7-12 $\mathrm{dB}$ and allows signals from gold nanorods corresponding to specific bindings to be distinguished. This approach potentially allows the expression levels of different oncogenes of cancer cells to be revealed simultaneously.
\end{abstract}

\section{INTRODUCTION}

C ANCER cells present altered surface molecules to en$\mathcal{C}$ courage their growth and metastasis, and the expressed oncogenic surface molecules are important in the prediction of clinical outcome and treatment response of anticancer drugs. Therefore, imaging cancer lesions while simultaneously obtaining information at a molecular level on the pathogenicity of as many oncogenic proteins as possible is of great clinical significance. To achieve this goal, we have explored the potential of photoacoustic (PA) imaging using bioconjugated gold nanorods as molecular probes for multiple selective targeting.

PA imaging generally involves irradiating an object with a laser pulse, which results in a local temperature rise due to absorption of optical energy, the induction of a

Manuscript received October 26, 2006; accepted March 23, 2007. Financial supports from National Science Council (grant\# NSC 952218-E-002-036), National Health Research Institutes, NTU Center for Genomic Medicine, and NTU Nano Center for Science and Technology are gratefully acknowledged.

P.-C. $\mathrm{Li}$ is with the Institute of Biomedical Electronics and Bioinformatics, National Taiwan University, Taipei, Taiwan (e-mail: paichi@cc.ee.ntu.edu.tw).

C.-W. Wei, and C.-K. Liao, and P.-C. Li are with the Department of Electrical Engineering, National Taiwan University, Taipei, Taiwan.

C.-D. Chen, K.-C. Pao, and C.-R. C. Wang are with the Department of Chemistry and Biochemistry, National Chung-Cheng University, Chiayi, Taiwan.

Y.-N. Wu and D.-B. Shieh are with the Institute of Molecular Medicine, National Cheng Kung University, Tainan, Taiwan.

Digital Object Identifier 10.1109/TUFFC.2007.435 temperature gradient, and the generation of the acoustic signals that are subsequently detected [1], [2]. PA imaging has been applied to several biomedical applications, including breast tumor detection [3], epidermal melanin measurements [4], and blood oxygenation monitoring [5]. Noninvasive structural and functional in vivo imaging of the rat brain also has been demonstrated [6]. The studies on PA imaging reported in the literature have mostly focused on the anatomical and functional levels. In contrast, the present study extended PA imaging to the molecular level with the capabilities of multiple, selective targeting. The hypothesis was that pathogenic information at the molecular level of many oncogenic proteins can be obtained simultaneously by PA imaging using bioconjugated gold nanoparticles as molecular probes.

Gold nanoparticles have been used for various biomedical applications [7]-[14], including as contrast agents for PA imaging [15], [16]. One intriguing property of gold nanoparticles is that their absorption spectra are geometry dependent. Size effects on the surface plasmon absorption of spherical gold nanoparticles result in the peak optical absorption red shifting with increasing particle size, with gold nanospheres with diameters of tens of nanometers exhibiting an absorption peak at about $550 \mathrm{~nm}$ [17]. In contrast, the wavelength of peak absorption of gold nanorods (i.e., cylindrical gold nanoparticles) depends only weakly on their diameter, instead increasing strongly with the aspect ratio (defined as the ratio of the length of the major axis to that of the minor axis). Laser irradiation at the wavelength corresponding to the peak absorption results in the maximal absorption cross section and thus the maximal PA signal.

Targeting gold nanoparticles at cancer cells helps in the early detection using PA imaging due to the large difference in the optical absorption between gold nanoparticles and the tissue [10]. In addition, targeting different molecules with bioconjugated gold nanorods having different aspect ratios also can be achieved. In this case, the antibodies are first conjugated to the surface of the gold nanorods. The bioconjugated gold nanorods interact with cells that have antigens specific to the conjugated antibody; hence, the cells that bind the bioconjugated gold nanorods can be detected using PA techniques. In this study, multiple targeting using gold nanorods with different aspect ratios was tested experimentally to demonstrate the feasibility of measuring the expression levels of different oncogenes of cancer cells simultaneously. The antiHER2 (human epidermal growth factor receptor 2) antibody is associated with growth characteristics and is sensitive to Herceptin chemotherapy. The anti-CXCR4 (CXCchemokine receptor 4) antibody is related to the prediction of the organ-specific metastatic potential of cancer cells for advanced clinical interventions. These two types 
of antibody were conjugated to nanorods with two aspect ratios.

Multiple selective targeting can be beneficial in two scenarios: to obtain the molecular profile of tumor cells reflecting the aggressiveness, metastatic potential, and chemotherapy drug resistance of the cells, which can be used to develop an optimal treatment strategy; and to identify the existence of a heterogeneous population of tumor cells in the same lesion. Our proposed approach is applicable to both of these scenarios. This study used two types of cell (MBT2 and HepG2), each with a specific antigen-antibody pair.

\section{Bioconjugation And TARgeting}

The MBT2 (murine bladder cancer) cell line was maintained in 90\% DMEM (Dulbecco's modified Eagle's medium) and 10\% FBS (fetal bovine serum), and the MBT2 KD (knock-down) cell line (MBT2 cell line with anti-HER2/neu knockout cell line) was cultured with the same media. The human hepatoma HepG2 cell line was cultured with $90 \%$ MEM (minimum essential medium Eagle), $2 \mathrm{mM}$ L-glutamine, $0.1 \mathrm{mM}$ nonessential amino acids, $1 \mathrm{mM}$ sodium pyruvate, and $10 \%$ FBS. The HepG2-N1 is a stable clone that is transfected with the GFP (green fluorescent protein) gene, and was further selected with $0.4 \mathrm{mg} / \mathrm{mL} \mathrm{G418.} \mathrm{All} \mathrm{of} \mathrm{the} \mathrm{cell} \mathrm{lines} \mathrm{were} \mathrm{maintained} \mathrm{in} \mathrm{an}$ incubator at $37^{\circ} \mathrm{C}$ with a humidified atmosphere containing $5 \% \mathrm{CO}_{2}$ in air. The medium was changed every 2 days. To subculture the cells before reaching growth confluence, the cells were washed twice with $10 \mathrm{~mL}$ of PBS (phosphatebuffered saline) per T75 flask to remove residual medium, then trypsinized using $3 \mathrm{~mL}$ of $0.25 \%$ trypsin/EDTA, followed by termination of the reaction by adding $3 \mathrm{~mL}$ of culture medium containing $10 \%$ FBS. The cells were centrifuged at $1000 \mathrm{rpm}$ for 10 minutes, and the supernatant was resuspended with culture medium and reseeded to another two T75 flasks or a Petri dish after counting the number of cells. The concentration of the cells was maintained at about $2.5 \times 10^{7}$ cells $/ \mathrm{mL}$ throughout this study.

The anti-HER2 antibodies were purified from the AHER2 hybridoma (CRL-10463), which was purchased from the American Type Culture Collection (Manassas, VA). The antibody production and purification were performed by GlycoNex (Taipei, Taiwan). Briefly, adjuvant primed adult male BALB/c mice (Laboratory Animal Center, NCKU, Tainan, Taiwan) were injected with hybridoma cells, and the mouse antibodies were then purified from mouse ascites on a protein A column and dialyzed in PBS.

Our primary goal was to prepare two types of molecular probe: $\mathrm{AuNR}_{785}-\mathrm{HER} 2$ and $\mathrm{AuNR}_{1000}-\mathrm{CXCR} 4$, in which the subscripts represent the peak absorption wavelengths (in nanometers) of gold nanorods with mean aspect ratios of 3.7 and 5.9, respectively, and "HER2" and "CXCR4" indicate the antibodies conjugated to the gold nanorods. In this study, the MBT-WT (wild type) cells and human hepatoma HepG2 cells overexpressed anti-HER2 and

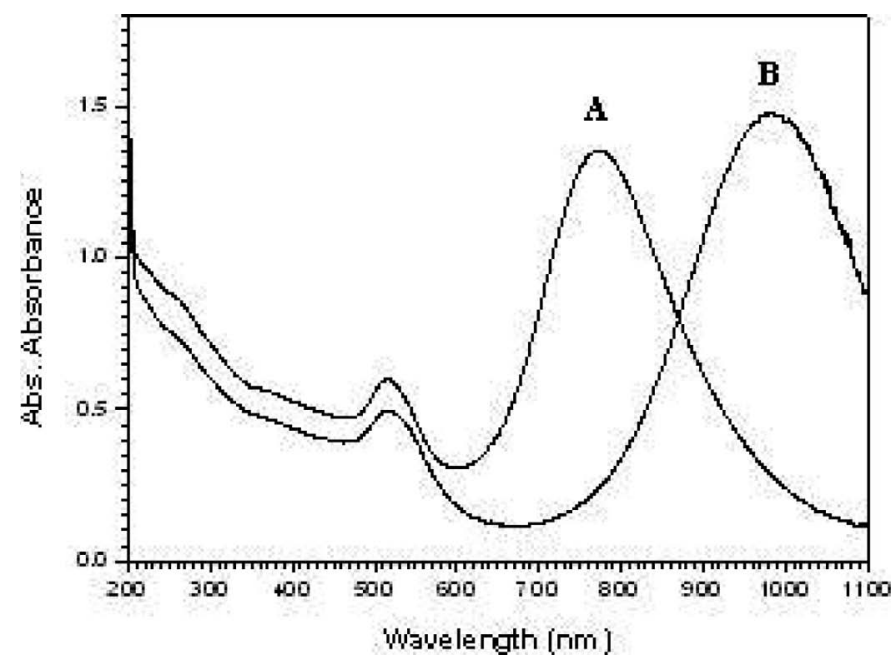

Fig. 1. Absorption spectra of molecular probes AuNR $785-\mathrm{HER} 2$ (A) and AuNR $1000-\mathrm{CXCR} 4$ (B). Absorbances of the spectra are adjusted for easy comparison.

anti-CXCR4 on the cell surface, respectively. The transmembrane glycoprotein anti-HER2 (p185/neu, c-ErbB-2) was found to be overexpressed in breast and ovarian cancer cells [18], whereas CXCR4 functions as a coreceptor for human immunodeficiency virus (HIV) and is considered an attractive target for the development of anti-HIV drugs [19].

Gold nanorods suspended in an aqueous solution were synthesized using an electrochemical method [20], then stabilized by the cationic surfactant molecules tetradodecylammonium bromide and hexadecyltrimethylammonium bromide. The two types of gold nanorod with distinct aspect-ratio distributions were synthesized to give longitudinal surface plasmon absorption bands located at 785 and $1000 \mathrm{~nm}$. The excess amount of surfactant molecules was removed by a flocculation and redispersion sequence. The aqueous solution containing resuspended gold nanorods then was ready for subsequent antibody conjugation and cell binding. The absorption spectra of the developed probes are shown in Fig. 1. The aqueous nanoparticle solutions were stored at $4^{\circ} \mathrm{C}$, and remained in a good dispersed condition for at least 1 month.

The particle shape was effectively controlled by the cationic cosurfactant micelles, which included several other ingredients such as cyclohexane and acetone. The particle mean aspect ratio was controlled by the release of a minor amount of silver ions and their releasing rate during electrolysis. This is done by immersing a silver metal plate into the electrolytic solution with two different specific immersing areas. Less surface area of the silver metal plate exposing in the solution generates gold nanorods with a smaller mean aspect ratio. This is believed to be associated with the small amount of silver ions released consume Br- of the micellar counter ions, and then the subsequent instability of the micelles causes preferential, one-dimensional growth of gold nanorods.

To evaluate the binding efficiency of an anti-HER2 antibody immobilized on the nanorod, the MBT2 cells were 


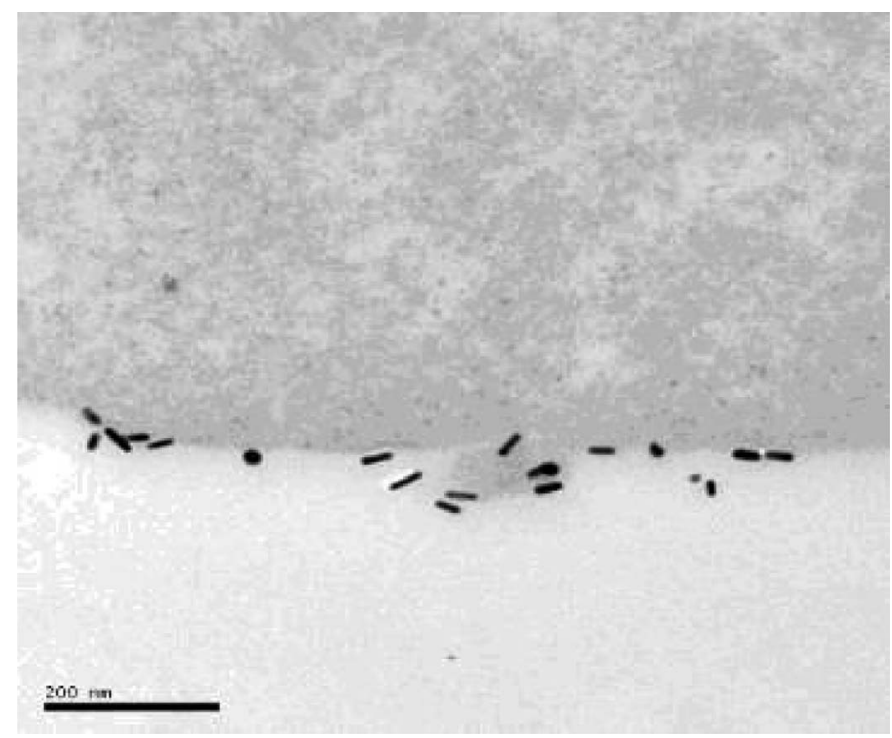

Fig. 2. Electron micrograph of a portion of an MBT2 cell targeted with AuNR 785 -HER2, clearly showing the presence of the gold nanorods (black cylinders) around the cell surface.

suspended by Versene (Gibco, Invitrogen, Carlsbad, CA), which contains $0.2 \mathrm{~g} / \mathrm{L}$ EDTA $4 \mathrm{Na}$ in PBS. The suspended cells were centrifuged at $1200 \mathrm{rpm}$ for 5 minutes. The supernatant was discarded, and the number of cells was adjusted to $1.2 \times 10^{6}$ in a microcentrifuge tube (SSI Technologies, Edmond, UK) and further resuspended in $50 \mu \mathrm{L}$ of PBS containing 10\% FBS plus $50 \mu \mathrm{L}$ of anti-HER2- or anti-CXCR4-antibody-conjugated nanorods at a concentration to produce an optical density of 1.2 and an absorption peak of 785 or $1000 \mathrm{~nm}$. The microcentrifuge tubes were shaken at $4^{\circ} \mathrm{C}$ for 1 hour. After the incubation, the unbound nanorods were removed by washing them with PBS and centrifuging them three times at $2850 \mathrm{rpm}$.

Prior to PA measurements, the efficacy of the binding between cells and the bioconjugated gold nanorods was examined using transmission electron microscopy (TEM; JEM 2010, JEOL, Tokyo, Japan) at $200 \mathrm{kV}$. Fig. 2 shows a TEM image of a 70-nm-thick MBT2 cell slice, which shows bioconjugated gold nanorods attached to the cell surface. Moreover, the specific binding of bioconjugated gold nanorods was demonstrated by the binding of HepG2 cells with $\mathrm{AuNR}_{1000}$-CXCR4. The number of gold nanorods targeting each cell was estimated by multiplying the mean number of gold nanorods $\left(n_{\mathrm{Au}}\right)$ counted in three slices by the number of slices $\left(N_{\text {slice }}\right)$ required for a single cell. The number of slices was estimated by dividing the size of the cell $\left(R_{\text {cell }}\right)$ by the slice thickness. For MBT2 cells, the number of slices was approximately $100\left(R_{\text {cell }}=7 \mu \mathrm{m}\right.$ divided by the slice thickness of $70 \mathrm{~nm}$ ). Therefore, the estimated number of gold nanorods attached on one cell was 5000 $\left(n_{\mathrm{Au}}=50\right)$. For HepG2 cells, the estimated number of gold nanorods was about $1400\left(R_{\text {cell }}=10 \mu \mathrm{m}\right.$ and $\left.n_{\mathrm{Au}}=10\right)$.

The targeting efficiency also was verified by Western blot analysis (protein identification based on both antibody reactions and antigens). Fig. 3 shows the Western

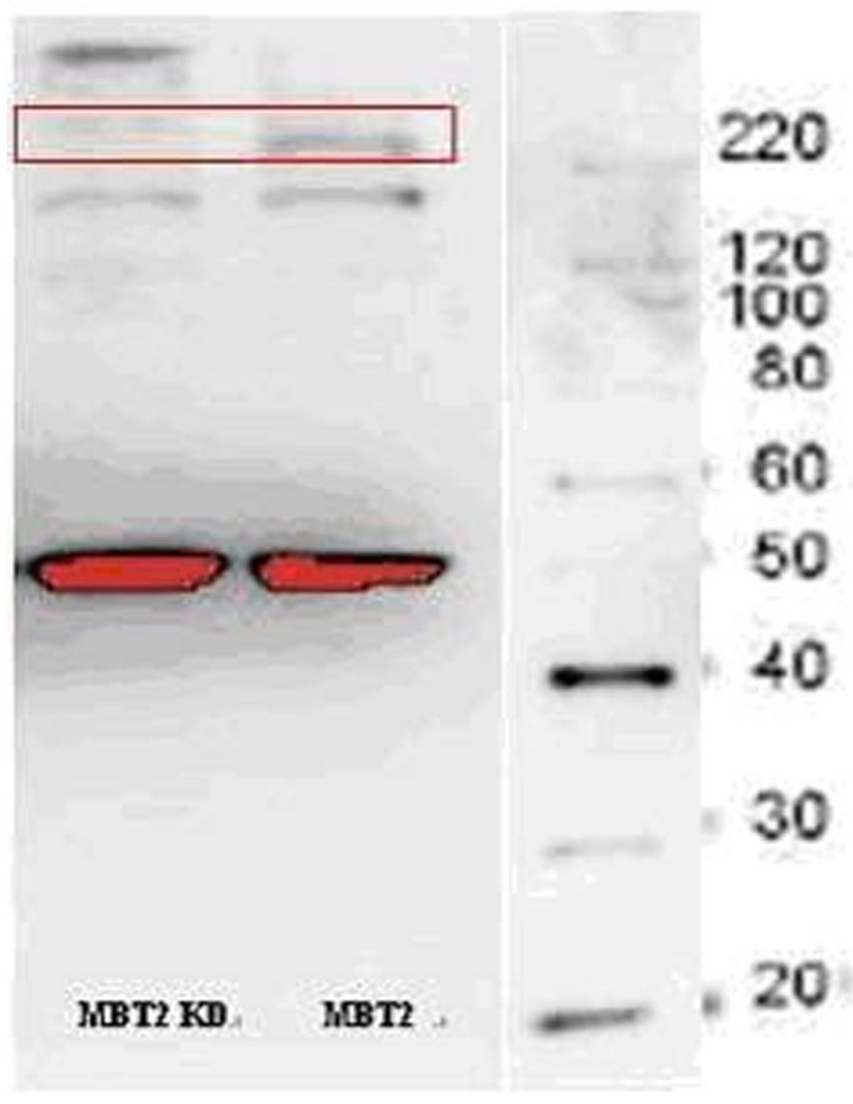

Fig. 3. (color online) Western blot analysis of MBT2 cells and MBT2 KD cells using anti-HER2 and actin proteins. The molecular weight of anti-HER2 is about 185,000 daltons; that of actin (as a reference) is about 50,000 daltons. The box shows the difference in the qualitative expression between MBT2 KD cells and MBT2 cells.

blot results of MBT2 KD cells and MBT2 cells using antiHER2, which exhibited high expression in MBT2 cells. A qualitative difference in the expression between MBT2 KD cells and MBT2 cells was evident near the 220,000 daltons marker (the molecular mass of anti-HER2 is about 185,000 daltons), indicating the specific targeting of anti-HER2 to MBT2 cells. The high expressions in both MBT2 KD cells and MBT2 cells at about 50,000 daltons were taken as a reference using actin protein, which exhibits high expression in most cells. The expression intensity ratios of antiHER2 to actin were $15.19 \%$ and $100 \%$ in MBT2 KD cells and MBT2 cells, respectively. Hence, the specific targeting of anti-HER2 to MBT2 cells was demonstrated.

\section{PA Imaging with Multiple Selective TARGETING}

Fig. 4 shows a schematic of the PA experimental setup. The samples were placed in a holder constructed from a transparent plastic material (Rexolite 1422, San Diego Plastics, San Diego, CA). The holder had external dimensions of $8 \times 4 \times 4 \mathrm{~cm}$ and contained two tubes with an internal diameter of $2.5 \mathrm{~mm}$. A wavelength-tunable pulsed 


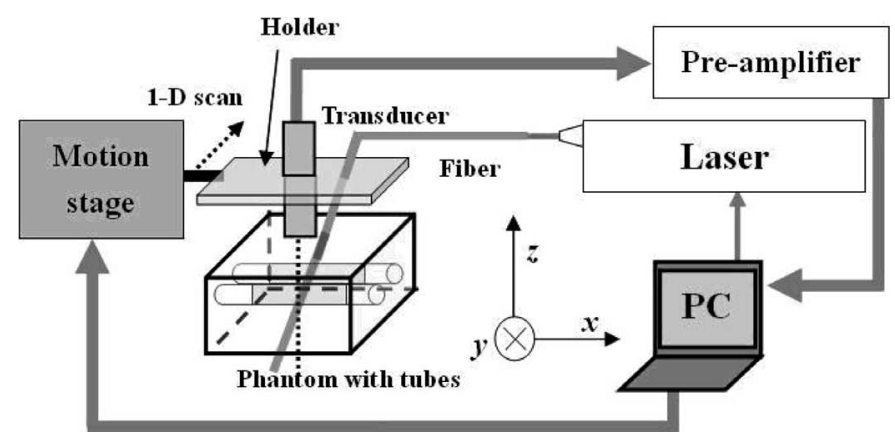

Fig. 4. Schematic of the experimental setup.

Ti-Sapphire laser (CF-125, SOLAR TII, Minsk, Republic of Belarus) with a pulse duration of $16 \mathrm{~ns}$ was used for optical excitation at 800 and $940 \mathrm{~nm}$, which approximately coincided with the peak absorption wavelengths of the two types of gold nanorod. The pulse repetition rate was $10 \mathrm{~Hz}$. The laser beam was coupled to a $1000-\mu \mathrm{m}$ fiber (FT-1.0-UMT, Thorlabs, Newton, NJ) to produce a round irradiation area with a diameter of about $3 \mathrm{~mm}$. The laser energy density at the two wavelengths was adjusted to be the same $\left(28.3 \mathrm{~mJ} / \mathrm{cm}^{2}\right)$. A single-crystal ultrasound transducer (V303, Panametrics, Waltham, MA) with a center frequency of $1 \mathrm{MHz}$, a diameter of $12.7 \mathrm{~mm}$, and a focal depth of $18 \mathrm{~mm}$ was used for PA signal detection (f-number $=1.4$ ). The fiber and the transducer were fixed in a holder that was driven by a precision motion stage (HR8, Nanomotion, Yokneam, Israel ) controlled by a motion controller (DMC-1842, Galil Motion Control, Rocklin, CA) housed in a personal computer to perform a onedimensional image scan. The acoustic waveforms were amplified (5072PR, Panametrics, Waltham, MA), then sampled by a 12-bit data acquisition card (CompuScope 12100, Gage, Lachine, QC, Canada) at 100 Msamples/s.

The efficacy of using bioconjugated gold nanorods to image multiple targets was assessed by separately testing the two types of cell (MBT2 and HepG2). PA images of the samples of pure MBT2 cells and MBT2 cells targeted with $\mathrm{AuNR}_{785}$-HER2 were acquired at both 800 and $940 \mathrm{~nm}$, as were those of pure HepG2 cells and HepG2 cells targeted with AuNR 1000 -CXCR4. The cell concentration in all four samples was $2.5 \times 10^{7}$ cells $/ \mathrm{mL}$. The top row of Fig. 5 shows a cross-sectional view of the sample placement in the tubes. The samples of cells targeted with bioconjugated gold nanorods were placed in the left tube, and those of the same cell type without targeting were placed in the right tube as a reference. The average intensities of image pixels in a $1.7 \times 1.3-\mathrm{mm}$ area within the tube were calculated.

The second row and the third row of Fig. 5 show images of MBT2 cells targeted with $\mathrm{AuNR}_{785}$-HER2 and pure MBT2 cells for laser irradiation at 800 and $940 \mathrm{~nm}$, respectively. The PA gray scale images were generated by taking an envelope of the received radio frequency data, and converting the envelope into $\log$ scale. The data acquired at each scanning position form a vertical line in the image. Dynamic range also is shown on the gray bar at the right of

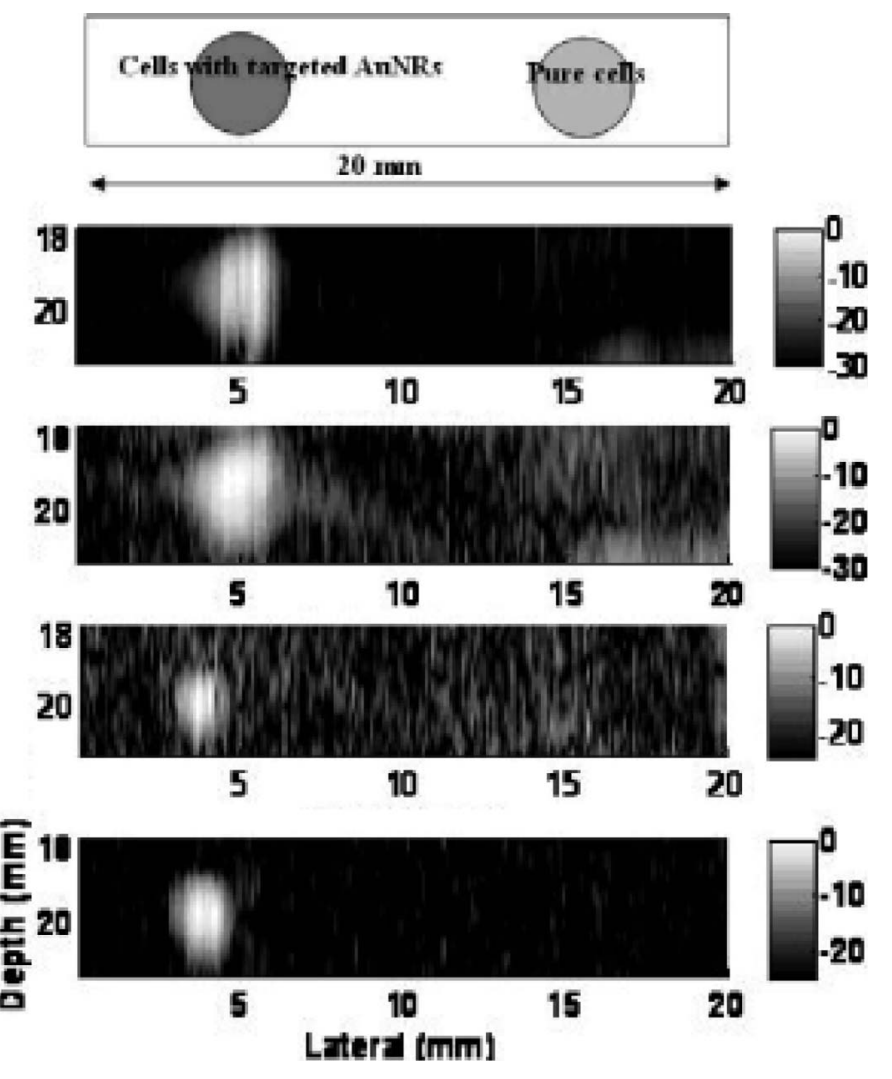

Fig. 5. (Top row) Cross-section schematic of sample placement in the tubes for PA measurements (for reference use with the lower four rows). AuNRs, gold nanorods. (Second row) Image of MBT2 cells targeted with AuNR 785 -HER2 (left) and pure MBT2 cells (right) for laser irradiation at $800 \mathrm{~nm}$ (in decibels). (Third row) Image of MBT2 cells targeted with AuNR 785 -HER2 (left) and pure MBT2 cells (right) for laser irradiation at $940 \mathrm{~nm}$ (in decibels). (Fourth row) Image of HepG2 cells targeted with AuNR $_{1000}$-CXCR4 (left) and pure HepG2 cells (right) for laser irradiation at $800 \mathrm{~nm}$ (in decibels). (Bottom row) Image of HepG2 cells targeted with AuNR $1000-$ CXCR4 (left) and pure HepG2 cells (right) for laser irradiation at $940 \mathrm{~nm}$ (in decibels).

the images. Note that, due to the limited spatial resolution of the imaging system, the images show intensities of the combined PA signals from all objects (gold nanorods and cells), instead of those from a single nanorod or a single cell.

For laser irradiation at 800 and $940 \mathrm{~nm}$, the average intensities were 25.02 and $17.29 \mathrm{~dB}$ higher, respectively, in the images of MBT2 cells with AuNR 785 -HER2 than in those of the pure MBT2 cells.

The fourth row and the bottom row of Fig. 5 show images of HepG2 cells targeted with AuNR $1000^{-C X C R} 4$ and pure HepG2 cells for laser irradiation at 800 and $940 \mathrm{~nm}$, respectively. The two images show opposite behavior to those of MBT2-HER2 bindings, in that the average intensities at 800 and $940 \mathrm{~nm}$ differed by 11.4 and $24.21 \mathrm{~dB}$, respectively, between the images of HepG2 cells with $\mathrm{AuNR}_{1000}-\mathrm{CXCR} 4$ and those of pure HepG2 cells. This comparison demonstrates the efficacy of the targeting of AuNR $1000-\mathrm{CXCR} 4$ to HepG2 cells using PA imaging. 


\section{Discussion}

The targeting effects evaluated by TEM and PA imaging were compared. Assuming that the measured PA intensity is linearly related to the concentration of gold nanorods (as verified in [21]), the concentration of the bioconjugated gold nanorods binding to cells can be calculated, and the number of bioconjugated gold nanorods per cell can be estimated. For example, the intensity of MBT2 cells targeted with AuNR 785 -HER2 was $17.71 \mathrm{~dB}$ lower than that of $\mathrm{AuNR}_{785}$-HER2 for a concentration of $1.45 \mathrm{nM}$ at $800 \mathrm{~nm}$. Hence, the concentration of bioconjugated gold nanorods binding to MBT2 cells can be estimated as:

$$
\frac{1.45 \mathrm{nM}}{17.71 \mathrm{~dB}}=\frac{1.45 \mathrm{nM}}{768}=0.19 \mathrm{nM}
$$

from which the number of bioconjugated gold nanorods per cell is:

$$
\begin{aligned}
\frac{0.19 \mathrm{nM}}{2.5 \times 10^{7 \mathrm{cells}} / \mathrm{mL}} & =\frac{1.14 \times 10^{14} \mathrm{AuNRs} / \mathrm{L}}{2.5 \times 10^{10 \mathrm{cells} / \mathrm{L}}} \\
& \approx 4550 \mathrm{AuNRs} / \mathrm{cell},
\end{aligned}
$$

which is close to the estimated result of 5000 gold nanorods per cell obtained from TEM.

This estimation also can be performed for HepG2 cells. The intensity of HepG2 cells targeted with AuNR $_{1000^{-}}$ CXCR4 was $15.87 \mathrm{~dB}$ lower than that of $\mathrm{AuNR}_{1000^{-}}$ CXCR4 for a concentration of $0.38 \mathrm{nM}$ at $940 \mathrm{~nm}$. Again, according to (1) and (2), the number of bioconjugated gold nanorods is estimated at 1472 per cell, which also is close to the estimated result of 1400 gold nanorods per cell obtained from TEM.

There is a tradeoff between binding as many gold nanorods as possible onto the target cell and avoiding nanorod aggregation. The aggregation potentially alters the absorption properties of gold nanoparticles, including the peak absorption wavelength and the bandwidth. There also is another tradeoff between antibody concentration and affinity of nanorods to the target cell. Although high antibody number per rod promotes binding affinity to the target cell due to multivalent effect, it also results in a lower number of nanorods on a cell given that the total antigen number is fixed. Therefore, the concentration applied at each step was experimentally adjusted to find the optimal tradeoff.

\section{CONClusions}

This study demonstrates the efficacy of using gold nanorods for applying PA molecular imaging to multiple targets. Targeting of MBT2 and HepG2 cells improved the average image contrast by 7.73 and $12.79 \mathrm{~dB}$, respectively. Further improvements in image contrast can be expected when gold nanorods can be produced with narrower aspect-ratio distributions. Moreover, this would allow the dual-targeting capability demonstrated in this study to be extended to targeting more than two types of objects. Future work also should focus on combining photothermal therapy with PA molecular imaging, whereby photothermal therapy techniques are developed for cancer treatment using the local heating from targeted gold nanoparticles under laser irradiation.

\section{REFERENCES}

[1] V. E. Gusev and A. A. Karabutov, "Laser optoacoustics," in Qualitative Theory of Thermooptical Sound Excitation in a Liquid. New York: American Institute of Physics, 1993, pp. 4-8.

[2] A. A. Karabutov, N. B. Podymova, and V. S. Letokhov, "Timeresolved laser optoacoustic tomography of inhomogeneous media," Appl. Phys. B, vol. 63, no. 6, pp. 545-563, 1996.

[3] R. O. Esenaliev, A. A. Karabutov, and A. A. Oraevsky, "Sensitivity of laser opto-acoustic imaging in detection of small deeply embedded tumors," IEEE J. Select. Topics Quantum Electron., vol. 5, no. 4, pp. 981-988, 1999.

[4] J. A. Viator, L. O. Svaasand, G. Aguilar, B. Choi, and J. S, Nelson, "Photoacoustic measurement of epidermal melanin," in Proc. SPIE, 2003, pp. 14-20.

[5] R. O. Esenaliev, I. V. Larina, K. V. Larin, D. J. Deyo, M. Motamedi, and D. S. Prough, "Optoacoustic technique for noninvasive monitoring of blood oxygenation: A feasibility study," Appl. Opt., vol. 41, no. 22, pp. 4722-4731, 2002.

[6] X. Wang, Y. Pang, G. Ku, X. Xie, G. Stoica, and L. V. Wang, "Noninvasive laser-induced photoacoustic tomography for structural and functional in vivo imaging of the brain," Natl. Biotechnol., vol. 21, no. 7, pp. 803-806, 2003.

[7] C. M. Niemeyer, "Nanoparticles, proteins, and nucleic acids: Biotechnology meets materials science," Angew. Chem. Int. Ed., vol. 40, pp. 4128-4158, 2001.

[8] C. C. Lin, Y. C. Yeh, C. Y. Yang, C. L. Chen, G. F. Chen, C. C. Chen, and Y. C. Wu, "Selective binding of mannoseencapsulated gold nanoparticles to type 1 pili," J. Amer. Chem. Soc., vol. 124, pp. 3508-3509, 2002.

[9] M. A. Hayat, Colloid Gold: Principles, Methods and Applications. New York: Academic, 1989.

[10] J. Kreuter, Microcapsules and Nanoparticles in Medicine and Pharmacy. Boca Raton, FL: CRC, 1992.

[11] A. P. Alivisatos, K. P. Johnsson, X. Peng, T. E. Wilson, C. J. Loweth, M. P. Bruchez, Jr., and P. G. Schultz, "Organization of nanocrystal molecules using DNA," Nature, vol. 382, pp. 609$611,1996$.

[12] C. A. Mirkin, R. L. Letsinger, R. C. Mucic, and J. J. Storhoff, "A DNA-based method for rationally assembling nanoparticles into macroscopic materials," Nature, vol. 382, pp. 607-609, 1996.

[13] T. A. Taton, C. A. Mirkin, and R. L. Letsinger, "Scanometric DNA array detection with nanoparticle probes," Science, vol. 289, pp. 1757-1760, 2000.

[14] A. K. Boal, F. Ilhan, J. E. DeRouchey, T. Thurn-Albrecht, T. P. Russell, and V. M. Rotello, "Self-assembly of nanoparticle into structured spherical and network aggregates," Nature, vol. 404, pp. $746-748,2000$.

[15] P.-C. Li, S.-W. Huang, C.-W. Wei, Y.-C. Chiou, C.-D. Chen, and C.-R. C. Wang, "Photoacoustic flow measurements by use of laser-induced shape transitions of gold nanorods," Opt. Lett., vol. 30, pp. 3341-3343, 2005.

[16] M. A. Eghtedari, J. A. Copland, V. L. Popov, N. A. Kotov, M. Motamedi, and A. A. Oraevsky, "Bioconjugated gold nanoparticles as a contrast agent for optoacoustic detection of small tumors," in Proc. SPIE, 2003, pp. 76-85.

[17] S. Link and M. A. El-Sayed, "Spectral properties and relaxation dynamics for surface plasmon electronic oscillations in gold and silver nanodots and nanorods," J. Phys. Chem. B, vol. 103, pp. 8410-8426, 1999.

[18] A. C. Steffen, M. Wikman, V. Tolmachev, G. P. Adams, F. Y. Nilsson, S. Stahl, and J. Carlson, "In vitro characterization of a bivalent anti-HER-2 affibody with potential for radionuclidebased diagnostics," Cancer Biother. Radiopharm., vol. 20, pp. 239-248, 2005. 
[19] S. Hatse, K. Princen, E. De Clercq, M. M. Rosenkilde, T. W. Schwartz, P. E. Hernandez-Abad, R. T. Skerlj, G. J. Bridger, and D. Schols, "AMD3465, a monomacrocyclic CXCR4 antagonist and potent HIV entry inhibitor," Biochem. Pharmacol., vol. 70, no. 5, pp. 752-761, 2005.

[20] S.-S. Chang, C.-W. Shih, C.-D. Chen, W.-C. Lai, and C.-R. C. Wang, "The shape transition of gold nanorods," Langmuir, vol. 15, no. 3, pp. 701-709, 1999.

[21] P.-C. Li, C.-W. Wei, C.-K. Liao, H.-C. Tseng, Y.-P. Lin, and C.-C. Chen, "Multiple targeting in photoacoustic imaging using bioconjugated gold nanorods," in Proc. SPIE, 2006, pp. 63-72. 